\title{
ИССЛЕДОВАНИЕ ПРОЦЕССА СТРУЙНОГО РАЗМЫВА ДОННЫХ ОТЛОЖЕНИЙ В НЕФТЯНЫХ РЕЗЕРВУАРАХ
}

\author{
Бутов Владимир Григорьевич',
} bvg@niipmm.tsu.ru

Никульчиков Андрей Викторович', nikulchikov@sibmail.com

\section{Никульчиков Виктор Кенсоринович', nikulchikov@tpu.ru}

\section{Солоненко Виктор Александрович², vik@niipmm.tsu.ru}

\author{
Ящук Алексей Александрович², \\ rainbow@niipmm.tsu.ru
1 Национальный исследовательский Томский политехнический университет, Россия, 634050, г. Томск, пр. Ленина, 30. \\ 2 Национальный исследовательский Томский государственный университет, \\ Россия, 634050, г. Томск, пр. Ленина, 36.
} Актуальность. При эксплуатации нефтяных резервуаров часто возникает проблема отложения твердых частиц и тяжелых пара-
финистых осадков на днище резервуара. Выпадение данных осадков влечет за собой уменьшение полезного объема резервуа-
ра, препятствует свободному перемешиванию слоев нефти, что в свою очередь способствует концентрации агрессивных раство-
ров солей в районе днища и развитию коррозионных разрушений нефтяных резервуаров. Для предотвращения подобных си-
туаций предусмотрен комплекс мер (например, ручная и механизированная очистка резервуаров сиспользованием горячей во-
ды и химических реагентов), однако существенный интерес представляют устройства, препятствююие выпадению донных
осадков без остановки эксплуатации резервуара, например винтовые устройства, размывающие донные отложения с помощью
турбулентной струи.
Цель исследования: расчет гидродинамических характеристик турбулентной затопленной струи в резервуаре при различных
температурах нефти; определение реологических параметров нефти, при которых возможно размытие донных отложений в резервуаре.

Объекты исследования: конструкция винтового устройства размыва донных отложений в резервуарах с нефтью, влияние основных режимных и конструктивных характеристик винта устройства на параметры турбулентной струи, моделирование процесса гидродинамического течения нефти в замкнутом объеме резервуара.

Методы: метод конечных объемов с использованием ANSYS CFX

В результате расчетов конструкций показаны зависимость скорости размыва от расстояния до винта для разных значений температур и соответствующей им вязкости нефти, показаны условия, при которых возможен размыв донных отложений в резервуаре для данной геометрии гребного винта.

\section{Ключевые слова:}

Нефть, реологическая модель, метод конечных объемов,

касательные напряжения, продольная скорость, скорость размыва.

\section{Введение}

При эксплуатации нефтяных резервуаров часто возникает проблема отложения твердых частиц и тяжелых парафинистых осадков на днище резервуара [1]. Выпадение данных осадков влечет за собой уменьшение полезного объема резервуара, препятствует свободному перемешиванию слоев нефти, что в свою очередь способствует концентрации агрессивных растворов солей в районе днища и развитию коррозионных разрушений нефтяных резервуаров [2-4]. Для предотвращения подобных ситуаций предусмотрен комплекс мер (например, ручная и механизированная очистка резервуаров с использованием горячей воды и химических реагентов [5-10]), однако существенный интерес представляют устройства, препятствующие выпадению донных осадков без остановки эксплуатации резервуара, например винтовые устройства, размывающие донные отложения с помощью турбулентной струи [11-13].

При работе устройства размыва при вращении лопастей гребного винта создается направленная затопленная турбулентная струя жидкости. Под воздействием затопленной турбулентной струи от лопастей винта нефть в резервуаре перемешивается, имеющиеся донные отложения размываются. Устройство для размыва донных отложений для интенсификации процесса перемешивания выполнено с возможностью поворота вала винта на угол $60^{\circ}$. При работе устройства в крайних точках в ре- 
зервуаре возникает круговое движение всей массы нефти в резервуаре.

Цель работы:

1) расчет гидродинамических характеристик турбулентной затопленной струи в резервуаре при различных температурах нефти;

2) определение реологических параметров нефти, при которых возможно размытие донных отложений в резервуаре.

\section{Методы}

При проведении расчетов трехмерной задачи применялся метод конечных объемов с использованием программного комплекса ANSYS CFX. Уравнения модели течения (1)-(5), реализованные в комплексе ANSYS CFX, представлены в руководствах по гидродинамике, например, в [14-17].

В рамках этого комплекса были построены конечно-объемные модели $[18,19]$, воспроизводящие геометрию рассматриваемых расчетных областей и позволяющие проводить комплексные расчеты с учетом пространственного турбулентного характера течения.

Для моделирования была использована конечно-объемная расчетная сетка с 1134867 элементами и 510450 узлами для расчета течения в резервуаре. Для моделирования течения в окрестности винта была использована сетка с числом элементов и узлов 394627 и 168245 соответственно.

Для описания турбулентного течения были применены эйлеровы переменные $x_{i}, i=1,2,3$. Течение в данной точке пространства во времени характеризуется для жидкости вектором скорости $\vec{U}$ с компонентами $u_{i}(i=1,2,3)$ и давлением $p$. Температура $T$ и плотность жидкости $\rho$ считаются постоянными величинами.

Уравнение неразрывности для осредненных по времени величин:

$$
\frac{\partial \rho}{\partial t}+\frac{\partial}{\partial x_{k}}\left(\rho u_{k}\right)=0
$$

Уравнение сохранения импульса:

$$
\frac{\partial\left(\rho u_{i}\right)}{\partial t}+\frac{\partial}{\partial x_{k}}\left(\rho u_{i} u_{k}\right)=-\frac{\partial p}{\partial x_{i}}+g_{i}+\frac{\partial \tau_{i k}}{\partial x_{k}},
$$

где $g_{i}$ - массовая сила в $i$-м направлении, в случае расчета $g_{1}=g_{2}=0, g_{3}=\rho g, g-$ ускорение силы тяжести.

Тензор вязких напряжений без учета влияния градиентов давления имеет вид

$$
\tau_{i j}=\mu\left(\frac{\partial u_{i}}{\partial x_{j}}+\frac{\partial u_{j}}{\partial x_{i}}\right)-\frac{2}{3} \mu \delta_{i j} \frac{\partial u_{k}}{\partial x_{k}},
$$

где $\mu$ - коэффициент вязкости, зависящий от температуры; $\delta_{i j}$ - символ Кронекера.

Уравнение для энергии турбулентного перемешивания:

$$
\begin{aligned}
& \frac{\partial(\rho k)}{\partial t}+\frac{\partial}{\partial x_{i}}\left(\rho u_{i} k\right)=\frac{\partial}{\partial x_{j}}\left(u_{i} \tau_{i j}\right)- \\
& -\beta^{*} \rho \omega k+\frac{\partial}{\partial x_{i}}\left(\left(\mu+\sigma^{*} \mu_{T}\right) \frac{\partial k}{\partial x_{i}}\right) .
\end{aligned}
$$

Уравнение для удельной скорости диссипации:

$$
\begin{gathered}
\frac{\partial(\rho \omega)}{\partial t}+\frac{\partial}{\partial x_{i}}\left(\rho u_{i} \omega\right)=\frac{\gamma \omega}{k} \frac{\partial}{\partial x_{j}}\left(u_{i} \tau_{i j}\right)- \\
-\beta \rho \omega^{2}+\frac{\partial}{\partial x_{i}}\left(\left(\mu+\sigma^{*} \mu_{T}\right) \frac{\partial k}{\partial x_{i}}\right) .
\end{gathered}
$$

Здесь $k$ - кинетическая энергия турбулентности; $\omega$ - частота турбулентных пульсаций.

Характеристики нефти при различных температурах приняты согласно [20].

Нефть имеет ньютоновские свойства при температуре свыше $20{ }^{\circ} \mathrm{C}$ включительно. Поскольку нефть имеет неньютоновские свойства при температуре ниже $20^{\circ} \mathrm{C}$, для ее описания при этих значениях использовалась модель вязкости Бингама-Шведова [20].

Геометрические и режимные параметры устройства задавались следующим образом:

- диаметр винта, мм - 690 (определялся размерами люка резервуара);

- количество лопастей винта, шт - 3;

- частота вращения винта, об/мин - 460;

- угол поворота вала винта, угл. град, не менее 60 . Расчеты проведены в диапазоне температур нефти от +10 до $+40^{\circ} \mathrm{C}$.

Вязкость нефти задавалась из диапазона $5-100$ сCт

В расчетах использовался нефтяной резервуар РВСП:

- объем, $\mathrm{M}^{3}-50000$;

- диаметр, м-60,7;

- высота стенки, м-18,1.

\section{Обсуждение полученных результатов}

Моделирование поведения затопленной турбулентной струи, формируемой винтом

Результаты моделирования турбулентной струи, формируемой винтом, с использованием полной 3-D модели винта при высоких температурах нефти (выше $20{ }^{\circ} \mathrm{C}$ ) представлены на рис. 1.

При частоте вращения винта 460 об/мин продольная скорость непосредственно за винтом составляет до $4,9 \mathrm{~m} / \mathrm{c}$ (рис. $1, \mathrm{~A})$.

В случае низких температур (менее $15{ }^{\circ} \mathrm{C}$ ) нефть проявляет неньютоновские свойства, результаты аналогичного расчета для этого случая представлены на рис. 2.

На рис. 3 приведена зависимость скорости размыва от расстояния до винта для разных значений 

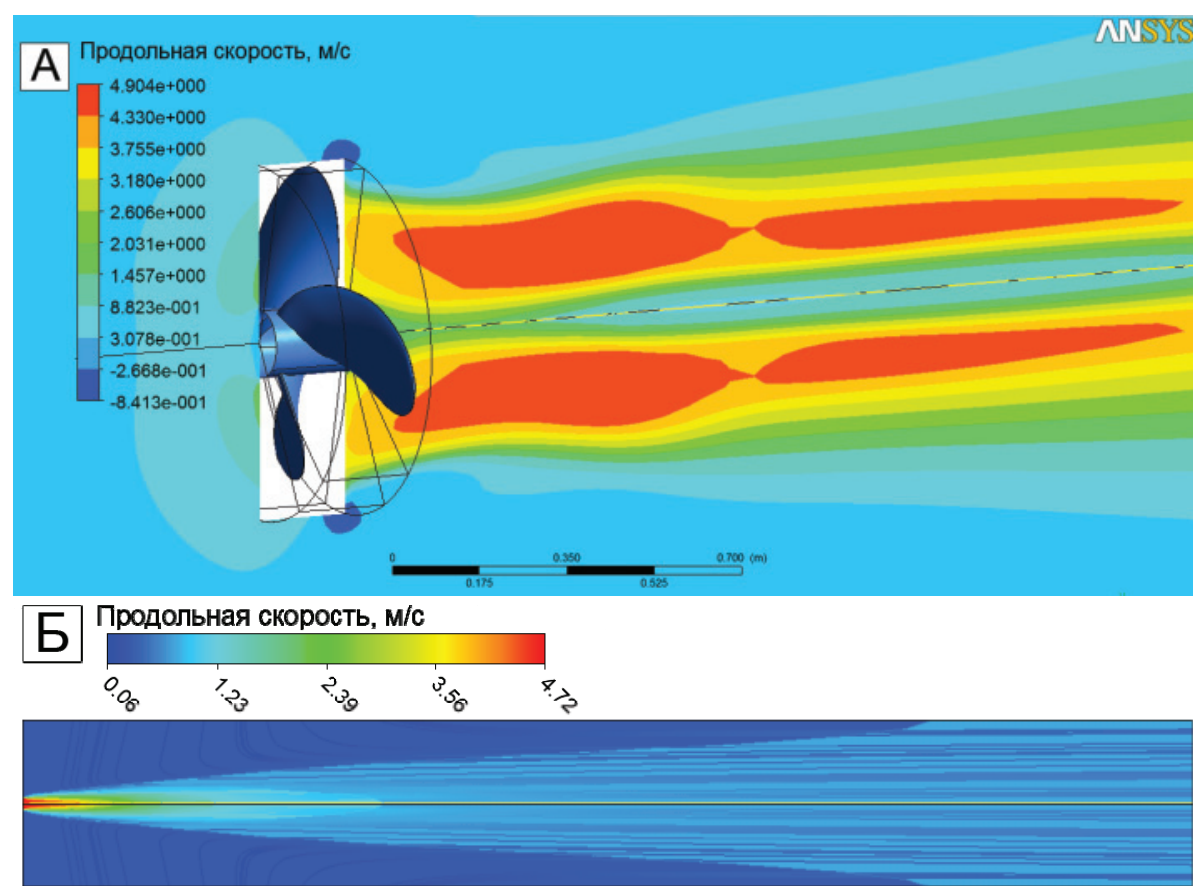

Рис. 1. Распределение продольной скорости при вращении винта с частотой 460 об/мин в нефти, подчиняющейся закону Ньютона в диапазоне температур свыше $20^{\circ} \mathrm{C}:$ в локальной окрестности винта (А) и в неограниченном (справа) пространстве (Б)

Fig. 1. Distribution of axial velocity in Newtonian oil within the temperature range higher than $20^{\circ} \mathrm{C}$ at propeller rotation frequency 460 rpm: in propeller vicinity (A); in unrestricted (on the right) space ( $(5)$

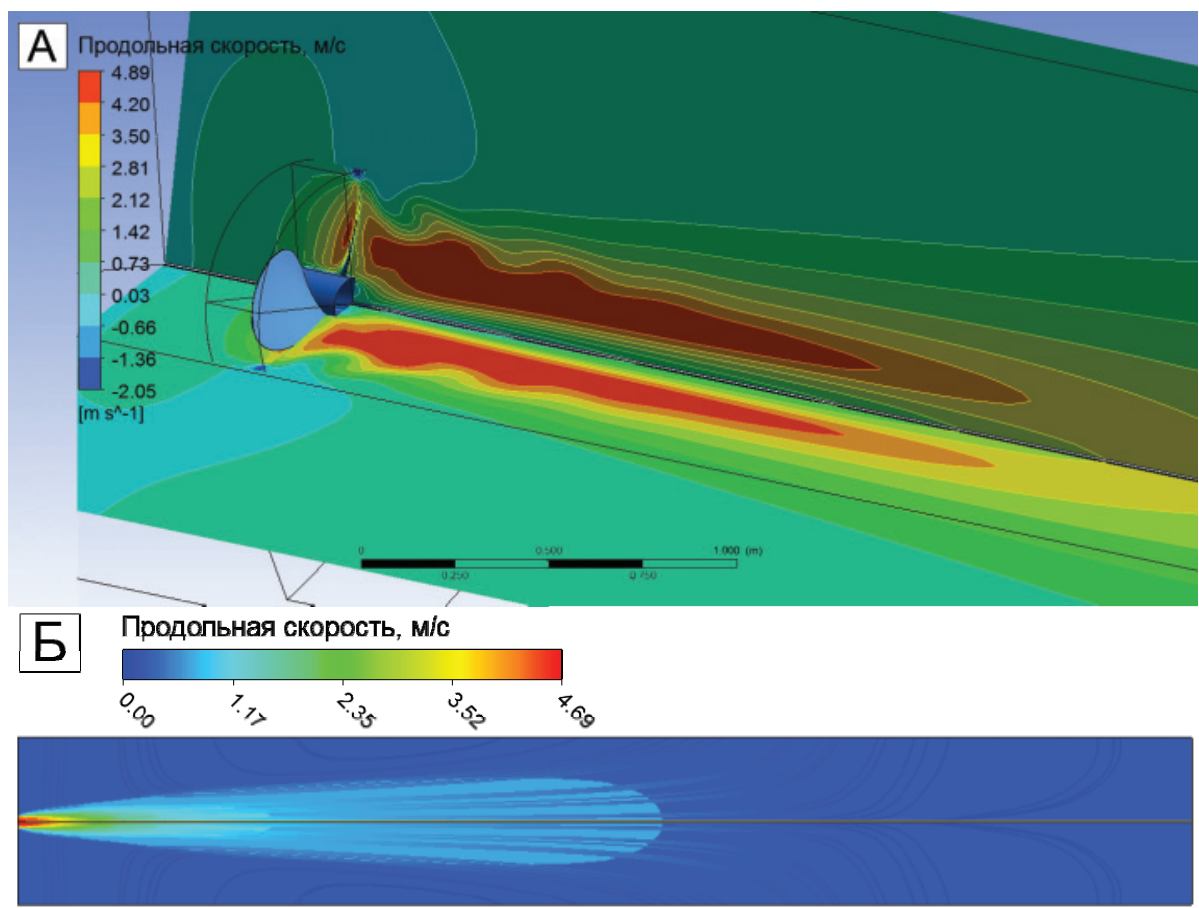

Рис. 2. Распределение продольной скорости при вращении винта с частотой 460 об/мин в нефти, подчиняющейся неньютоновскому закону при температуре $10{ }^{\circ} \mathrm{C}:$ в локальной окрестности винта (А) и в неограниченном (справа) пространстве (Б)

Fig. 2. Distribution of axial velocity in oil that exhibits non-Newtonian characteristics at temperature $10{ }^{\circ} \mathrm{C}$ and propeller rotation frequency $460 \mathrm{rpm}$ : in propeller vicinity $(A)$; in unrestricted (on the right) space (Б) 
температур и соответствующих им вязкостей нефти. Из результатов видно, что в случае соответствия модели течения ньютоновскому приближению скорость размыва на расстоянии 60,7 м от винта в случае неограниченного объема нефти будет составлять $0,6 \mathrm{~m} / \mathrm{c}$ при вязкости, большей 30 сСт.

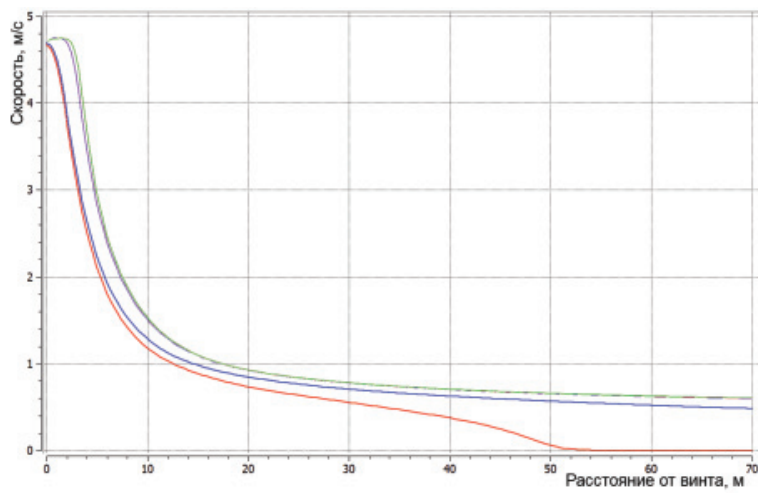

Рис. 3. Зависимость скорости размыва от расстояния до винта для разных значений температур и соответ ствующих им вязкостей: сиреневому цвету соответствует температура $20{ }^{\circ} \mathrm{C}$ или вязкость 30 сСт (ньютоновская модель); зеленому цвету соответствует температура $40^{\circ} \mathrm{C}$ или вязкость 9 сСт (ньютоновская модель); синему цвету соответствует температура $15^{\circ} \mathrm{C}$ или вязкость 50 сСт (неньютоновская модель); красному цвету соответствует температура $10{ }^{\circ} \mathrm{C}$ или вяз кость 100 сСт (неньютоновская модель)

Fig. 3. Dependence of resuspension velocity on the distance from the propeller at various temperatures and corresponding oil viscosities: magenta color indicates temperature $20^{\circ} \mathrm{C}$ or viscosity $30 \mathrm{CSt}$ (Newtonian model); green color indicates temperature $40{ }^{\circ} \mathrm{C}$ or viscosity $9 \mathrm{CSt}$ (Newtonian model); blue color indicates temperature $15{ }^{\circ} \mathrm{C}$ or viscosity 50 cSt (non-Newtonian model); red color indicates temperature $10^{\circ} \mathrm{C}$ or viscosity $100 \mathrm{cSt}$ (nonNewtonian model)

\section{Моделирование течения в резервуаре}

При моделировании течения внутри ограниченного объема при наличии одного устройства размыва донных отложений при варьировании поворота этого устройства в пределах $\pm 30^{\circ}$ от направления на центр резервуара область, где скорость струи позволит осуществить размыв, будет представлена следующими рисунками.

На рис. 4 различными цветами представлены результаты определения области размыва при различных температурах и соответствующих им вязкостях. Графически скорость размыва в зависимости от расстояния до винта для различных температур и соответствующих им вязкостей показана на рис. 5. На рис. 6 представлены линии тока в объеме резервуара и профили скорости вдоль оси винта при нулевом отклонении оси винта для случая разных реологических моделей жидкости.

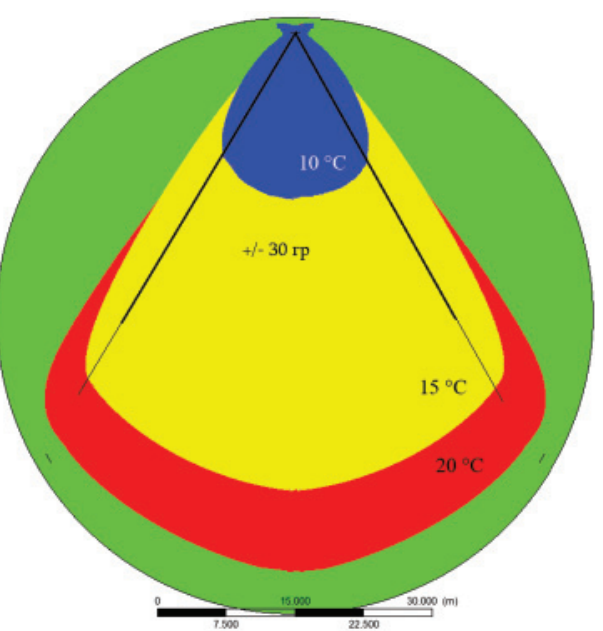

Рис. 4. Область размыва в зависимости от температуры (при 20,15 и $10^{\circ} \mathrm{C}$ )

Fig. 4. Resuspension zone for various temperatures (20, 15 and $\left.10{ }^{\circ} \mathrm{C}\right)$

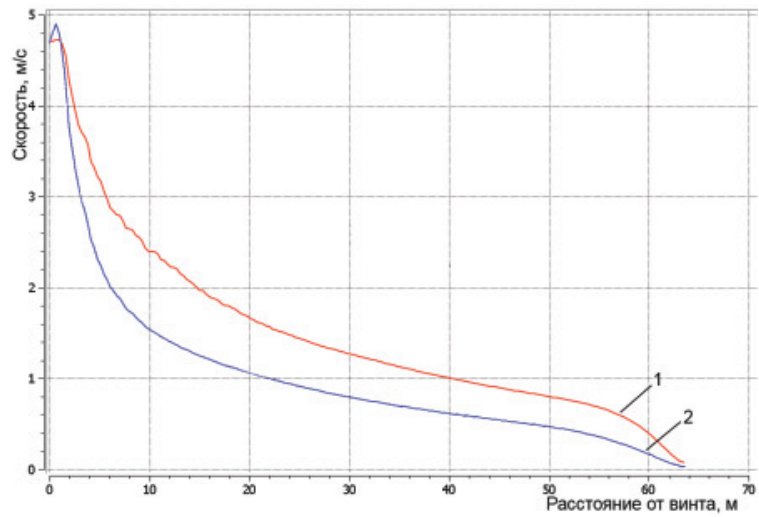

Рис. 5. Зависимость скорости размыва от расстояния до винта: 1 - при вязкости 30 сСт в резервуаре (в рамках ньютоновской реологической модели); 2 - при вязкости 50 сСт в резервуаре (в рамках неньютоновской реологической модели)

Fig. 5. Dependence of resuspension velocity on the distance from the propeller: 1 - at viscosity 30 CSt in tank (within Newtonian rheological model); 2 - at viscosity 50 CSt in tank (within non-Newtonian rheological model)

\section{Выводы}

По результатам моделирования можно сделать следующие выводы:

1) при заданных параметрах гребного винта при температурах нефти свыше $20{ }^{\circ} \mathrm{C}$ и указанного диапазона вязкости нефти возможно формирование потока жидкости со скоростью 0,5 м/с на расстоянии 60,7 м от лопастей винта в случае неограниченного объема нефти;

2) при температуре нефти ниже $15^{\circ} \mathrm{C}$ для достижения требуемых параметров в случае неограни- 

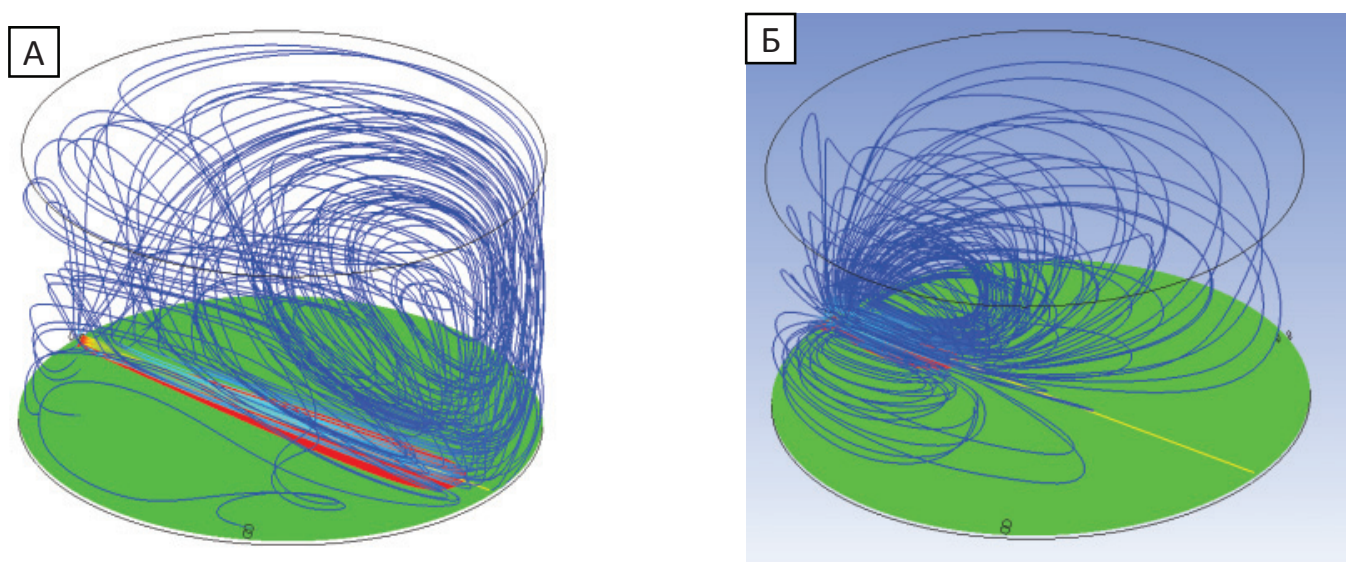

Рис. 6. Линии тока для вязкости 30 ССт в рамках ньютоновской реологической модели (А) и 100 сСт в рамках неньютоновской реологической модели (Б)

Fig. 6. Lines of flow with viscosity 30 cSt within Newtonian rheological model (A) and 100 cSt within non-Newtonian rheological model (5)

ченного объема нефти размыва достичь не удается (скорость 0,5 м/с на расстоянии 60,7 м от лопастей винта);

3) при размыве донных отложений в ограниченном объеме (резервуаре) для устранения застойных зон, находящихся вблизи стенок резервуара, в обязательном порядке необходимо применение нескольких устройств, устано-

\section{СПИСОК ЛИТЕРАТУРЫ}

1. Characterization of the sludge deposits in crude oil storage tanks / I.A. Gopang, H. Mahar, A.S. Jatoi, K.S. Akhtar, M. Omer, M.S. Azeem // Journal of Faculty of Engineering \& Technology. - 2016. - V. 23 (1). - P. 57-64.

2. Рыбалко П.Н., Прибытков П.А., Бурков П.В. Исследование напряженно-деформированного состояния днища резервуара с коррозионным поражением // Проблемы геологии и освоения недр: Труды XVIII Международного симпозиума имени академика М.А. Усова студентов и молодых ученых - Томск: ТПУ, 2014. - T. II. - C. 624-625.

3. Гималетдинов Г.М., Саттарова Д.М. Способы очистки и предотвращения накопления донных отложений в резервуарах // Нефтегазовое дело. - 2006. - T. 1. URL: http://ogbus.ru/article/sposoby-ochistki-i-predotvrashheniya-nakopleniya-donnyxotlozhenij-v-rezervuarax (дата обращения 13.06.2018).

4. Experimental investigations of various methods of sludge measurements in storage oil tanks / M. Monteiro, V. Svet, D. Sandilands, S. Tsysar // Advances in Remote Sensing. - 2015. - V. 4. № 2. - P. 119-137.

5. Чурикова Л.А., Конашева Е.А., Утегалиев А.Т. Обзор современных методов очистки резервуаров от нефтяных остатков // Технические науки в России и за рубежом: материалы V Междунар. науч. конф. - М., январь 2016 г. - М.: Буки-Веди, 2016. - C. 71-75.

6. Hu G., Li J. Zeng G. Recent development in the treatment of oily sludge from petroleum industry: a review // Journal of hazardous materials. - 2013. - V. 261. - P. 470-490.

7. Method for removing sludge in crude oil tank and recovering oil therefrom: patent US6673231B2, United States, Publ. 06.01.2004. вленных симметрично по периметру первого яруса резервуара. Количество устройств размыва донных отложений зависит от объема резервуара, свойств нефти и может быть определено расчетом.

Исследования проводились в рамках выполнения государственного задания Министерства образования и науки России, проект № 9.9752.2017/8.9.

8. Method for cleaning storage tanks: patent US20070283981A1, United States, Publ. 13.12.2007.

9. Oil tank sludge removal method: patent US20060042661A1, United States, Publ. 2.03.2006.

10. Water washing method and system for crude oil tank: patent US20170291200A1, United States, Publ. 12.10.2017.

11. Костин М.В., Демин А.В., Садчиков А.В. Очистка емкостей от остатков нефти и нефтепродуктов с помощью эффлюэнта // Научное обозрение. Биологические науки. - 2015. - № 1. C. 93.

12. Чепур П.В., Тарасенко А.А. Особенности совместной работы резервуара и устройств размыва донных отложений винтового типа // Фундаментальные исследования. - 2015. - № 2. C. 1671-1675.

13. Nekrasov V.0. Modeling bottom sediment erosion process by swirling the flow by tangential supply of oil in the tank // IOP Conference Series: Materials Science and Engineering: Transport and Storage of Hydrocarbons. - 2016. - V. 154. 7 p. URL: http://iopscience.iop.org/article/10.1088/1757-899X/ 154/1/012003 (дата обращения 13.06.2018).

14. Флетчер К. Вычислительные методы в динамике жидкостей: В 2-х т. Т. 1. - М.: Мир, 1991. -504 с.

15. Андерсон Д., Таннехил Дж., Плетчер Р. Вычислительная гидромеханика и теплообмен. - М: Мир, 1990. - 726 с.

16. Уилкокс Д.К. Многомасштабная модель турбулентных течений // Аэрокосмическая техника. - 1989. - № 11. - С. 47-60.

17. Численное решение многомерных задач газовой динамики / под ред. С.К. Годунова. - М.: Наука, 1976. - 400 с.

18. Применение системы ANSYS к решению задач механики сплошной среды. Практическое руководство / под ред. проф. А.К. Любимова. - Нижний Новгород: Изд-во Нижегородского госуниверситета, 2006. - 227 с. 
19. Burkov P.V., Burkova S.P., Samigullin V.D. Computer Simulation of Stress-Strain State of Oil Gathering Pipeline Designed for Ugut Field // IOP Conference Series: Materials Science and Engineering. - 2016. - V. 125: Materials Treatment: Current Problems and Solutions. $-7 \mathrm{p}$.
20. Трубопроводный транспорт нефти и газа / Р.А. Алиев, В.Д. Белоусов, А.Г. Немудров, В.Л. Юфин, Г.И. Яковлев. - М.: Недра, 1988. - 368 c.

Поступила 15.06.2018 г.

\section{Информация об авторах}

Бутов В.Г., доктор физико-математических наук, профессор отделения ядерного топливного цикла Инженерной школы ядерных технологий Национального исследовательского Томского политехнического университета.

Никульчиков A.B., кандидат физико-математических наук, доцент отделения нефтегазового дела Инженерной школы природных ресурсов Национального исследовательского Томского политехнического университета.

Hикульчиков B.K., кандидат технических наук, доцент отделения нефтегазового дела Инженерной школы природных ресурсов Национального исследовательского Томского политехнического университета.

Солоненко B.A., кандидат физико-математических наук, заведующий лабораторией оптимизации отдела математической физики Научно-исследовательского института прикладной математики и механики Томского государственного университета.

$\boldsymbol{A}$ щук $\boldsymbol{A} . \boldsymbol{A}$., кандидат физико-математических наук, старший научный сотрудник лаборатории оптимизации отдела математической физики Научно-исследовательского института прикладной математики и механики Томского государственного университета. 


\title{
SIMULATION STUDY OF BOTTOM SEDIMENTS JET EROSION IN OIL TANK
}

Vladimir G. Butov,

bvg@niipmm.tsu.ru

Andrey V. Nikulchikov',

nikulchikov@sibmail.com

Viktor K. Nikulchikov',

nikulchikov@tpu.ru

Victor A. Solonenko ${ }^{2}$, vik@niipmm.tsu.ru

\author{
Alexey A. Yashchuk', \\ rainbow@niipmm.tsu.ru \\ 1 National Research Tomsk Polytechnic University, \\ 30, Lenin Avenue, Tomsk, 634050, Russia. \\ ${ }^{2}$ National Research Tomsk State University, \\ 36, Lenin Avenue, Tomsk, 634050, Russia.
}

The relevance. When crude oil is stored in tanks, invariably solid particles and heavy paraffin sediments are deposited. The precipitation of these sediments entails a decrease in reservoir useful volume, prevents free mixing of oil layers, which boosts the concentration of aggressive salt solutions in the bottom region and the development of corrosive destruction of oil reservoirs. A set of measures is envisaged to prevent such situations (including manual and mechanized cleaning of tanks using hot water and chemical reagents), however, the devices that prevent precipitation of bottom sediments without stopping the operation of the reservoir are of significant interest, for example, screw devices that dilute bottom sediments with the help of turbulent jet.

The aim of the research is calculation of hydrodynamic properties of turbulent submerged jet at various oil temperatures; definition of oil rheological parameters which enable the lift off, mix, and re-suspension of sediments in a tank.

Objects of research: design of the bottom sediment erosion device for oil tanks; influence of the screw regime and design parameters on the turbulent jet properties; simulation of oil hydrodynamic flow in a closed reservoir volume.

Methods: finite volume method in ANSYS CFX.

Results. The paper demonstrates the dependence of sediment resuspension velocity on the distance from the screw at various temperatures and corresponding oil viscosities. The conditions to re-suspend oil tank bottom sediments were defined for the given propeller geometry. It was proved that the screw-type device is an effective means to lift solids off the storage tank floor and ensure required characteristics of tank oil.

\section{Key words:}

Oil, rheological model, finite volume method, shear stress, longitudinal velocity, resuspension velocity.

The research was carried out within the State Task of the Ministry of Science and Education of the Russian Federation, project no. 9.9752.2017/8.9.

\section{REFERENCES}

1. Gopang I.A., Mahar H., Jatoi A.S., Akhtar K.S., Omer M., Azeem M.S. Characterization of the sludge deposits in crude oil storage tanks. Journal of Faculty of Engineering \& Technology, 2016, vol. 23 (1), pp. 57-64.

2. Rybalko P.N., Pribytkov P.A., Burkov P.V. Issledovanie naprya zhenno-deformirovannogo sostoyaniya dnishcha rezervuara [Study of stress-strain behavior of the corroded oil tank bottom surface]. Tomsk, TPU Publ., 2014, vol. 2, pp. 624-625.

3. Gimaletdinov G.M., Sattarova D.M. Sposoby ochistki i predotvrasheniya nakopleniya donnykh otlozheniy $v$ reservuarakh [Methods of cleaning and preventing accumulation of bottom sediments in oil tanks]. Neftegazovoe delo, 2006, vol. 1. Available at: http://ogbus.ru/article/sposoby-ochistki-i-predotvrashheniya-nakopleniya-donnyx-otlozhenij-v-rezervuarax (accessed 13 June 2018).
4. Monteiro M., Svet V., Sandilands D., Tsysar S. Experimental investigations of various methods of sludge measurements in storage oil tanks. Advances in Remote Sensing, 2015, vol. 4, no. 2, pp. 119-137.

5. Churikova L.A., Konasheva E.A., Utegaliev A.T. Obzor sovremennykh metodov ochistki reservuarov ot neftyanykh ostatkov [Modern methods of cleaning oil tanks: a review]. Tekhnicheskie nauki $v$ Rossii $i$ za rubezhom. Materialy $V$ Mezhdunarodnoy nauchnoy konferentsii [Technical sciences if Russia and abroad: Proc. of International scientific conference]. Moscow, January 2016. Moscow, Buki-vedi Publ., 2016, pp. 71-75.

6. Hu G., Li J., Zeng G. Recent development in treatment of oily sludge from petroleum industry: a review. Journal of hazardous materials, 2013, vol. 261, pp. 470-490.

7. Kim M.-J., Yoon D.-S., Choi C.-H., Choi J.-T., Kwak J.-W., Park S.-K. Method for removing sludge in crude oil tank and recovering oil therefrom. Patent United States, US6673231B2, 2004. 
8. Stewart T.E. Method for cleaning storage tanks. Patent United States, US20070283981A1, 2007.

9. Meyer D., Brons G., Perry R., Wildemeersch S., Kennedy R. Oil tank sludge removal method. Patent United States, US20060042661A1, 2006.

10. Son S.G. Water washing method and system for crude oil tank. Patent United States, US20170291200A1, 2017.

11. Kostin M.V., Demin A.V., Sadchikov A.V. Tank cleaning residues from oil and oil products through the effluent. Scientific review. Biological sciences, 2015, vol. 1, p. 93. In Rus.

12. Chepur P.V., Tarasenko A.A. The features of washout sediments devices screw type and tank collaboration. Fundamental research, 2015, vol. 2, pp. 1671-1675. In Rus.

13. Nekrasov V.0. Modeling bottom sediment erosion process by swirling the flow by tangential supply of oil in the tank. IOP Conference Series: Materials Science and Engineering: Transport and Storage of Hydrocarbons, 2016, vol. 154, 7 p. Available at: http://iopscience.iop.org/article/10.1088/1757-899X/ 154/1/012003 (accessed 13 June 2018).

14. Fletcher C.A.J. Computational Techniques for Fluid Dynamics. Berlin, Springer-Verlag, 1988. 409 p.
15. Anderson D.A., Tannehill J.C., Pletcher R.H.I. New York, McGraw-Hill, 1984, 609 p.

16. Wilcox D.C. Multiscale Model for Turbulent Flows. AIAA Journal, 1988, vol. 26, no. 11, pp. 1311-1320.

17. Chislennoe reshenie mnogomernykh zadach gazovoy dinamiki [Numerical solution of multidimensional problems in gas dynamics] Ed. by S.K. Godunov. Moscow, Nauka Publ., 1976, 400 p.

18. Primenenie sistemy ANSYS $k$ resheniyu zadach mekhaniki sploshnoy sredy. Prakticheskoe rukovodstvo [Use of ANSYS software in solving problems of continuum mechanics]. Ed. by A.K. Lyubimov. Nizhny Novgorod, Nizhny Novgorod State University Press, 2006, $227 \mathrm{p}$.

19. Burkov P.V., Burkova S. P., Samigullin V. D. Computer Simulation of Stress-Strain State of Oil Gathering Pipeline Designed for Ugut Field. IOP Conference Series: Materials Science and Engineering, 2016, vol. 125: Materials Treatment: Current Problems and Solutions, $7 \mathrm{p}$.

20. Aliev R.A., Belousov V.D., Nemudrov A.G., Truboprovodny transport nefti $i$ gaza [0il and gas pipeline transport]. Moscow, Nedra Publ., 1988, 368 p.

Received: 15 June 2018.

\section{Information about the authors}

Vladimir G. Butov, Dr. Sc., professor, National Research Tomsk Polytechnic University.

Andrey V. Nikulchikov, Cand. Sc., associate professor, National Research Tomsk Polytechnic University.

Viktor K. Nikulchikov, Cand. Sc., associate professor, National Research Tomsk Polytechnic University.

Victor A. Solonenko, Cand. Sc., head of laboratory, Tomsk State University.

Alexey A. Yashchuk, Cand. Sc., senior research assistant, Tomsk State University. 